\title{
Computational Model of Successful Mobilization of Voluntary Firefighters
}

\author{
Joze Srekl \\ Faculty of Chemistry and Chemical Technology, University of Ljubljana, Ljubljana, Slovenia \\ Email: joze.srekl@fkkt.uni-lj.si
}

Received 2 June 2013; revised 3 February 2014; accepted 15 February 2014

Copyright (C) 2014 by author and Scientific Research Publishing Inc.

This work is licensed under the Creative Commons Attribution International License (CC BY). http://creativecommons.org/licenses/by/4.0/

(c) (i) Open Access

\begin{abstract}
Reliability, the part of successful convocations of a voluntary fire brigade, is one of the decisive factors of successfulness and quality of a voluntary fire brigade. The unplanned selection and education of firemen show that the unit is not reliable enough. The presented mathematical model serves as a numerical evaluation of reliability. It helps to analyze the causes of eventual weak points and to make effective steps toward their improvement. This method is the first step to a large analysis of quality and reliability of a voluntary fire brigade. The calculations represent a mathematical model that is not fully equivalent to the real situation, but it depicts well enough and is much cheaper to find and test optimal solutions. It is also relatively easy to test the reliability of each voluntary fire brigade (VFB).
\end{abstract}

\section{Keywords}

Volunteer Firefighter; Effectiveness; Model of Calculation

\section{Introduction}

The volunteer fire brigades have more than one hundred years of tradition in central Europe. The professional fire service has emerged with the development of cities and industries. It has developed a parallel system of professional and volunteer units. A dual system is established in Slovenia and is required by law [1]. A similar system of fire-fighting units is known in almost all European countries including USA, Canada and Australia. In the United States it has been found that the cost of voluntary fire-fighters work is lower [2] but generally volunteer fire departments are less successful. Volunteer fire departments are entirely appropriate where the community is prepared for the longer response time, less frequent intervention. However, new trends threaten the survival of volunteer fire departments [3]-[5]. Simpson [5] noted that the volunteer fire department is much easier to organize in rural areas than in suburban and urban environments. 
A good qualification is the first condition for successful work of volunteer fire departments. Therefore, the law [1] sets minimum standards of competence of volunteer firefighters. Awareness of the importance of knowledge and skills for action is increasing among the firefighters. The success of the voluntary fire brigade (VFB) intervention depends not only on the competence of this unit, but also the possibility that at any given time a composed full unit will be able to intervene.

Members of the VFB are only volunteers who have their private lives, which fit the time available to intervene. In Slovenia they have no members of VFB in a duty of standby or partially employed, as is usual in some western European countries [6], so the time available is only random, unplanned and unexpected. It is impossible to talk about some of the available real time, just talking about the times when it is likely that the firefighter responds to a call that is relatively large. Therefore, it is also impossible to talk about some exact accounts, which very accurately characterizes the quality of the response of a VFB. With the help of probability and statistics, it is nevertheless possible to calculate certain parameters that are sufficiently likely to be taken into account when assessing the ability of VFB, or in the planning of more efficient units.

\section{Theoretical Background}

We must clearly define concepts and they prescribe a numeric value for the consistent development of the theory. The effectiveness of interventions VFB is a rather abstract concept, although it can be numerically evaluated.

Let:

$d(t)$ — estimated damage that occurred without the intervention of VFB,

$d(p)$ - the actual damage that has occurred despite the transmission of VFB,

Then we can define efficiency as the ratio:

$$
u=\frac{d(t)-d(p)}{d(t)}=1-\frac{d(p)}{d(t)}
$$

We immediately noted that the performance can determine following the intervention. The success of $u$ takes values between 0 and 1 . Collection team performance is a measure of quality of VFB after the intervention. This quality is dependent on the skills, technical equipment and reliability. Exists whole range of random factors which cannot be assumed and they influenced this result. Some of these factors deal with the theory of catastrophes. Our intention is to be a little more specific dealing with a single factor, namely the reliability of VBF. Reliability is defined as the ratio between success and all references to the unit for intervention.

Let:

$n$-Number of references to intervene,

$m$-Number of successful references (a sufficient number of members in good draw)

Then the reliability:

$$
z=\frac{m}{n}
$$

It takes values between 0 and 1 . This value can be determined based on the actual selection of interventions and determine the composition of the collected units. Number of interventions is not so much to be statistically reliable conclusions on this value. You could otherwise help with test alarms, but this way does not produce reliable results because the alarm would have to repeat too often and reduce the severity of response.

\section{Data Collection}

The first step in examining the reliability of one or more VFB is to collect the data necessary for constructing the model. All members will be interviewed VBE that we will know when they will call and when to reach the inaccessible. Of course, the interview, as usual, should be addressed in a standard form. Here you can decide for a variety of ways. We are not interested in a particular situation, but by an average state for many years, so that we formally divide the year at 53 weeks, we add another week to start the normal 52 weeks. Each week we split into two units: 5 working days of a single unit and a two day weekend (Saturday and Sunday) is the second unit. The day was divided into four parts, so we have a total of 424 data for each fireman.

Data was collected using questionnaires; responses are subject to the discretion of individual firefighters and their assessment of life in recent years. Because of random events, the facts may vary from the surveyed values. 
However, it is a way to get to a model that is sufficiently likely to be realized. The decision for such a scheme of data, as we said above, the result of the way of life for us, most normal patterns of life by living people (workers, farmers, partly as a staff) who have a voluntary fire brigade. If interviewed in an environment where the fire brigade are only farmers, or the time these people are different (not working in shifts, but in the "European" working time), could be decided on a different distribution of time intervals in the survey.

On the basis of a sufficiently large number of respondents, firefighters could be defined as models typifying firefighters as:

- Fireman-a worker in shifts (two or three)

- Fireman-farmer

- Fireman-the worker or employee in a single shift

- Fireman-students

Etc.

In addition to these elemental types can be defined as a mixture of types such as fireman-the farmer and the worker. The standardized types of fireman could have helped in constructing the optimal team with maximum reliability.

The collected data was properly processed. We know that a successful intervention unit is needed to meet three conditions. The unit must have at least one commander and deputy commander, also must carry at least one driver and a sufficient number of fire fighters.

Let:

$n(\min )$ - minimum number of firefighters needed to intervene,

$n(k)$-number of commanders or deputy

$n(v)$-number of drivers

$n(g)$-number of other firefighters

For the successful departure of the intervention inequalities must be met:

$$
\begin{aligned}
& n(k) \geq 1 \\
& n(v) \geq 1 \\
& n(k)+n(v)+n(g) \geq n(\min )
\end{aligned}
$$

This objective can be reached at any convening, and remain problematic, especially the first two inequalities. This would be corrected in the number of commanders, or deputy, and the number of drivers in the number of firefighters in the unit. The model will show us the current structure fire suitable for a reliable effect.

The processing of data from the survey put together three files. The first contains $n(k)$, the other $n(v)$ and the third $n(g)$ in all intervals of the year. It would be useful to keep all information the survey, but will have to study enough to start, if we have these three files.

\section{Calculation of Reliability}

Reliability is a value between 0 and 1 and is from the classical definition of probability of the event relationship between the number of favorable outcomes and the number of trials. This probability could be calculated on the basis of statistics of past events, but we note that there were too few events to reliably estimate the value.

In the previous section we created a file that gave value to inequalities (3), (4) and (5). In the individual time intervals from 1 to 424 establish the validity or invalidity of inequality constraints. Count all the positive results where inequalities (3), (4) and (5) are satisfied. By counting, we get a number of favorable outcomes $m$. Reliability estimated by equation (6).

$$
z=\frac{m}{424}
$$

In this assessment, we did not consider the time intervals of different lengths. We can use this calculation as a rough estimate of the reliability but it doesn't give us very accurate results.

We have decided to use the intervals of equal length in the next step. We opted for the concrete year to properly take into account the respective days in the year. All year, divided into hours, which means $n=365 \times 24$. In calculating $m$ is required for each date and time of first noted in any of 424 sites belonging to help determine the 
validity or invalidity of the inequality constraints (3) to (5). We are dividing the number of good hours with all hours of the year.

$$
z=\frac{m}{8760}
$$

The result, which is obtained in such a way, is sufficiently a reliable calculation of the probability of the classical definition of probability. Of course, the calculation does not take into account the uncertainty of the survey. It also gives us the result, for example, $z=0.87$ tells too little about the actual situation. Otherwise, for our example, we know that in a long sequence of intervention $87 \%$ of those where the team will be composed of complete and incomplete is $13 \%$ of the team. We are not interested in the effectiveness of WFB in a long statistical period, but only for effectiveness in a brief period. A short period, for example, one year, can be simulated only by the random method of selection events. This will somewhat blur the impact of a long process, which has a known result.

The Monte Carlo method-random selection of time intervals, intervention, interventions, selected annual sample. The sample size is random, also selected times. He decided for a number of samples. Among the selected samples of the sample, $n(i)$ selected times during the $m(i)$ favorable outcomes have security.

$$
z(i)=\frac{m(i)}{n(i)}
$$

Reliability of the unit is calculated using the average reliability of the samples.

$$
z=\frac{1}{k} \sum_{i=1}^{k} z(i)=\frac{1}{k} \sum_{i=1}^{k} \frac{m(i)}{n(i)}
$$

Also in this calculation, we must be careful. It is true that we at moderate sample size and not too many samples get a satisfactory result. The quality of the result could be checked only with the facts of interventions. If we use too many over-samples, we get the same result as in Equation (7). Effective is a simple argument, which links classical and statistical definition of probability, and is written in Equation (10).

$$
\lim _{k \rightarrow \infty} \frac{1}{k} \sum_{i=1}^{k} \frac{m(i)}{n(i)}=\frac{m}{n}
$$

\section{The Test of Model}

The model was tested on VFB in small local communities close to larger cities. The unit considered 26 people, including three commanders or deputy commander, five drivers and 18 base firefighters. All these people were tested and the results are stored in files. Table 1 shows some of the data between twenty-five and thirty-six time intervals during the year. The data shows that the full intervention group is assembled in 8 out of 12 cases. The problem is not in the number of firefighters as they were the minimum number that can always be provided (column G). The problem lies in providing a sufficient number of commanders or deputy. This means that the unit has enough suitably qualified senior firefighters.

Programs that process the data are written in FORTRAN. Treatment consists of several steps. The first step is to calculate the value of the Formula (2). It is therefore a classical probability calculation without taking into account the different length intervals. Note that the minimum number of firefighters in the inequalities (5) $n(\mathrm{~min})$ $=5$. This number could be even higher (at least 7) but does not significantly change the results. By computation we get the result for $m$ and $z$.

$$
m=347 \quad z=0.818
$$

In the next step we calculate the true value of probability, where we will present, as discussed, favorable outcome at equal intervals. The year is divided into $365 \times 24=8760$ hours. For each hour we look at how is the possibility of assembling a team. The result is different from the result (11) in the second decimal place.

$$
m=7238 \quad z=0.8363
$$

It is necessary to introduce additional parameters for the third step of computation. We have to decide how 
Table 1. The data between twenty-five and thirty-six time intervals during the year.

\begin{tabular}{lllll}
\hline $\mathbf{T}$ & $\mathbf{K}$ & $\mathbf{V}$ & $\mathbf{G}$ & $\mathbf{S}$ \\
\hline 25 & 1 & 2 & 6 & 9 \\
26 & 2 & 2 & 3 & 7 \\
27 & 1 & 4 & 13 & 18 \\
28 & 2 & 5 & 17 & 24 \\
$\mathbf{2 9}$ & $\mathbf{0}$ & $\mathbf{1}$ & $\mathbf{1 1}$ & $* *$ \\
$\mathbf{3 0}$ & $\mathbf{0}$ & $\mathbf{3}$ & $\mathbf{1 6}$ & $* *$ \\
31 & 1 & 4 & 14 & 19 \\
32 & 1 & 5 & 18 & 24 \\
$\mathbf{3 3}$ & $\mathbf{0}$ & $\mathbf{1}$ & $\mathbf{8}$ & $* *$ \\
$\mathbf{3 4}$ & $\mathbf{0}$ & $\mathbf{1}$ & $\mathbf{7}$ & $* *$ \\
35 & 2 & 3 & 15 & 20 \\
36 & 2 & 5 & 18 & 25 \\
\hline
\end{tabular}

T-Serial number of the interval; K-Number of commanders or deputy; V-Number of drivers; GNumber of ordinary firefighters; S-The total number if it is greater than or equal to 5.

large samples will allow and how many samples we took on the average. We decided that the maximum sample size of 50 trials, with sample size will be selected by random value. For the random sample size, we decided to totally ascertain purpose. Computer subroutines for generating random numbers do not really generate random numbers, but numbers are calculated using a function. Each outcome generates a new result that is almost random number. With any new start is generated the same "random" numbers. If we use random numbers in two directions, namely to make the sample selection and sample size, the randomness increases. Our intention is to simulate the situation with random events, which are otherwise much more than they will in fact happen. Thus we included the operation of the statistical laws of large number of experiments providing a sufficiently good average.

Calculation has shown that it is not important how many samples we take. A single sample is so large that it gives the average, which by increasing the number of samples will not change much. It is important to complete different conclusions. By randomly selecting a sample, the reliability decreases. We see that in such a manner defined reliability that comes closest to the actual situation, much lower than the conventional method calculated by the likelihood of reliable units. Let's look at how it changes the reliability of the number of samples.

Table 2 shows that the values of sample size with growth slowing and a value of $=0.62$. This is significantly lower than the value we calculated in (11) and (12).

\section{Conclusions}

We take into account social factors which affect the efficiency of units in VFB. The reliability of VPG, which is calculated by our method, is much lower than expected by calculation from the classical definition of probability. The model was also relatively easy to enter the coincidence of events. We see that this coincidence brings further uncertainty. The potential presence should increase at least $90 \%$, or perhaps to $95 \%$ in order to achieve sufficiently high reliability.

Collected data for all year show that it is practically meaningless by increasing the number of firefighters. Only five have such terms as the lack of available firefighters. The number of drivers is equivalent to very few periods when the drivers unit is not complete. The biggest problem is the insufficient number of firefighters, which may lead to intervention. Of course, this should also be in the education of new managers with caution. The network analysis would need to select a few firefighters that could look for a new commander or assistant. Thus, targeted education and promotion should result in greater reliability. Consideration in searching for new 
Table 2. Probability as a function of sample size ( $N$-sample size, $z$-probability).

\begin{tabular}{ll}
$N$ & $Z$ \\
\hline 1 & 0.7419 \\
21 & 0.5960 \\
41 & 0.6693 \\
61 & 0.5955 \\
81 & 0.6238 \\
101 & 0.6139 \\
121 & 0.6281 \\
141 & 0.6181 \\
161 & 0.6193 \\
181 & 0.6282 \\
201 & 0.6108 \\
221 & 0.6300 \\
241 & 0.6126 \\
261 & 0.6262 \\
281 & 0.6259 \\
\hline
\end{tabular}

firefighters should consider in advance their available time and look for people to fill the gaps in the incomplete formation teams. Just increasing the units would not significantly increase reliability.

\section{References}

[1] Zakon o gasilstvu (Uradno prečiščeno besedilo) (ZGas-UPB1) UR.L. RS, No. 113/2005.

[2] Brunet, A., De Boer, L. and McNamara, K. T. (2001) Community Choice between Volunteer and Professional Fire Departments. Nonprofit and Voluntary Sector Quarterly, 30, 26-50. http://dx.doi.org/10.1177/0899764001301002

[3] Perkins, K.B. (1987) Volunteer Fire Departments: Community Integration, Autonomy and Survival. Human Organization, 46, 342-348.

[4] Perkins, K.B. (1990) Volunteer Fire and Rescue Corporations: Structure, Process and Survival. Nonprofit and Voluntary Sector Quarterly, 19, 359. http://dx.doi.org/10.1177/089976409001900406

[5] Simpson, C. (1996) A Fraternity of Danger. American Journal of Economics and Sociology, 55, 17-34. http://dx.doi.org/10.1111/j.1536-7150.1996.tb02705.x

[6] Graham, J. (1992) A Comparative Study of Firefighting Arrangements in Britain, Denmark, the Netherlands and Sweden. Home Office Research Study, HMSO. 


\section{List of Symbols and Abbreviations}

$d(t)$ — estimated damage that occurred without the intervention of VFB,

$d(p)$ - the actual damage that has occurred despite the transmission of VFB

$u$-Efficiency as the ratio

$n$-Number of references to intervene

$m$-Number of successful references (a sufficient number of members in good draw)

z-Reliability

$n$ (min) - Minimum number of firefighters needed to intervene

$n(k)$-Number of commanders or deputy commanders

$n(v)$-Number of drivers

$n(g)$-Number of other firefighters

$\mathbf{T}$ - Serial number of the interval

$\mathbf{K}$-Number of commanders or deputy commanders

$\mathbf{V}$-Number of drivers

G-Number of ordinary firefighters

S-The total number if it is greater than or equal to 5

$N$-Sample size

VFB — voluntary fire brigade 